\title{
ON THE ALGEBRAIC CRITERIA FOR LOCAL PARETO OPTIMA. II
}

\author{
BY
}

\author{
YIEH-HEI WAN
}

\begin{abstract}
Constrained vector optimization problems in the large are studied in this paper. Fixing any constraints so that the feasible set is a manifold with corners, we prove that the set of local Pareto optima for typical vector-valued functions admit Whitney prestratifications. Furthermore, these prestratifications persist under small perturbation of vector-valued functions. The main tools used here are a variant of Thom's transversality theorem and the stratification theory of semialgebraic sets.
\end{abstract}

1. Introduction. Motivated by mathematical economics, we consider the problem of optimizing several functions with constraints simultaneously (cf. [6], [10], [11]). More precisely, let us fix a $C^{\mu}$ mapping $g=\left(g_{1}, \ldots, g_{c}\right)$ from a given smooth manifold $M^{n}$ into $R^{c}$. Denote by $W_{g}=\left\{x \in M^{n} \mid g_{1}(x) \geqslant\right.$ $\left.0, \ldots, g_{c}(x) \geqslant 0\right\}$. Now, let $f$ be any $C^{\mu}$ mapping from $M^{n}$ into $R^{p}$. A point $x \in W_{g}$ is said to be a local Pareto optimum (strict local Pareto optimum) for $f$ with inequality constraints $g$ if and only if there exists a neighborhood $N$ of $x$ such that for any $y$ in $N \cap W_{g} f_{i}(y) \geqslant f_{i}(x)$ for $i=1, \ldots, p$ implies $f_{i}(y)=$ $f_{i}(x)$ for $i=1, \ldots, p(y=x)$. The natural questions to ask are the following:

(A) Can one find certain necessary and sufficient conditions for local Pareto optima with given constraints for some generic class of $C^{\mu}$ mappings from $M^{n}$ into $R^{p}$ ?

(B) What is the generic picture of the set of local Pareto optima with given constraints for $C^{\mu}$ mappings from $M^{n}$ into $R^{p}$ ?

These questions are related to each other. If one can solve question (A) in a satisfactory way, then the answer to question (B) follows without too much difficulty. The second order conditions for local Pareto optima as introduced in [10] or [7] are not enough for the characterization of local Pareto optima when $n \geqslant p \geqslant 3$ (cf. [12]). In my previous work [11], I found a necessary and sufficient $\min (n, p)+1$ order algebraic condition for local Pareto optima without constraints for a generic class of mappings from $M^{n}$ into $R^{p}$. Thus, one could conclude that generically the set of local Pareto optima without constraints has a Whitney (pre) stratification. Furthermore when $M^{n}$ is

Received by the editors August 15, 1977 and, in revised form, October 13, 1977.

AMS (MOS) subject classifications (1970). Primary 58C99, $90 \mathrm{C} 99$.

Key words and phrases. Local Pareto optima, Whitney prestratification, semialgebraic sets, Thom's transversality theorem.

(c) American Mathematical Society 1979 
compact, this stratification is stable under small perturbations. The presence of constraints will complicate the optimization problem. This can be easily seen by the following simple example:

Let $M^{n}$ be the cartesian plane $R^{2}$. The constraint $g$ is given by $(x, 1-$ $x, y, 1-y)$. Thus, the feasible set $W_{g}$ is the unit square $[0,1] \times[0,1]$. Now we define $f$ to be a smooth map as in Diagram 1 which folds $R^{2}$ along a curve $\gamma$ passing through the points $b, e$ and maps the edge $\overparen{a b c}$ diffeomorphically onto the parabolic like arc $\widehat{f(z) f(b) f(c)}$, the arc $\overparen{b e}$ diffeomorphically onto the $\operatorname{arc} \widehat{f(b) f(e)}$, etc.
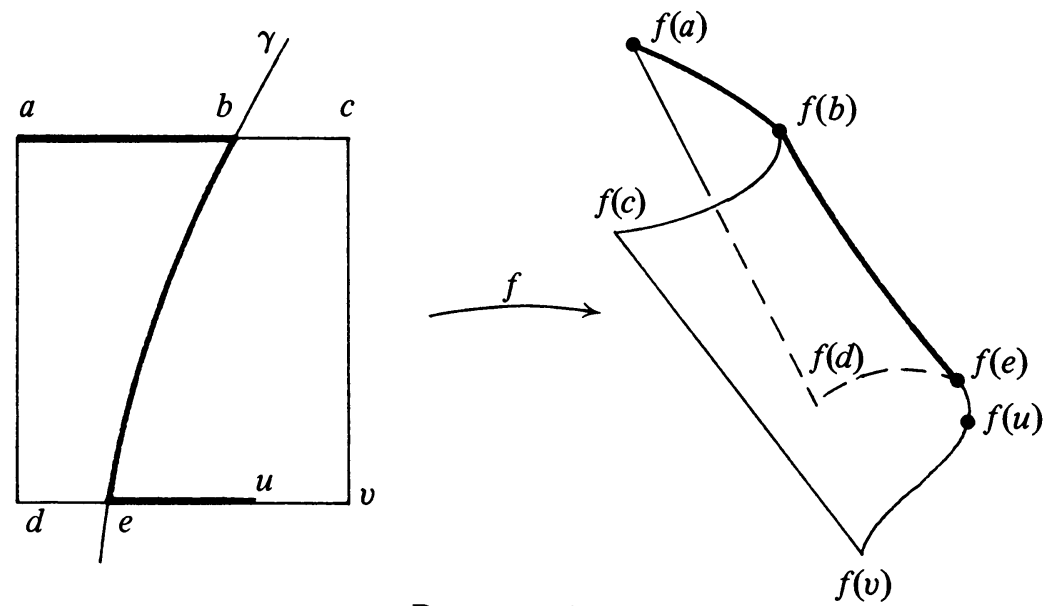

Diagram 1

It is clear from the picture that ignoring constraints, the set of local Pareto optima of $f$ lying in the square $[0,1] \times[0,1]$ is the arc $\widehat{b e}$. It is also clear that the set of local Pareto optima of $f$ with constraints $g$ is the union of the three $\operatorname{arcs} \widehat{a b}, \widehat{b e}, \widehat{e u}$. Hence the presence of the constraints $y \geqslant 0,1-y \geqslant 0$ adds two more arcs $\overparen{a b}, \overparen{e u}$ to the set of local Pareto optima of $f$. The purpose of this paper is to answer questions (A) and (B) under reasonable conditions on the constraints.

Set $N(x)=\left\{i \mid g_{i}(x)=0\right\}$ and $|N|=$ the cardinal number of the set $N(x)$. Write $g_{N(x)}=\left(g_{1}, \ldots, g_{i_{|N|}}\right): M^{n} \rightarrow R^{|N|}$ where $i_{1}<\cdots<i_{N}$ and $\left\{i_{1}, \ldots\right.$, $\left.i_{|N|}\right\}=N(x)$. We shall say that the constraints $g$ satisfy the regularity boundary condition if for any $x$ in $M^{n} g_{N(x)}: M^{n} \rightarrow R^{|N|}$ is of rank $|N|$ at $x$. From Thom's transversality theorem, we know that the set of constraints $g$ on $M^{n}$ which satisfy the regularity boundary condition forms an open dense subset in the space of $C^{\mu}$ mappings from $M^{n}$ into $R^{c}$. Under this assumption on the constraints $g$ the feasible set $W_{g}$ is either empty or a $n$ dimensional manifold with corners. The regularity boundary condition is the suitable assumption which will be made on the constraints throughout in our results.

In order to handle the high order criteria in a neat way, one is led to the 
notion of jets (cf. [2], §2 below). Denote by $J^{r}\left(n, R^{p+c}\right)=J^{r}\left(n, R^{p}\right) \times$ $J^{r}\left(n, R^{c}\right)$ the vector space of all $r$-jets of local $C^{r}$ mappings $(f, g):\left(R^{n}, 0\right) \rightarrow$ $R^{p} \times R^{c}$. Given $k=\left(k_{1}, \ldots, k_{c}\right) \in J^{r}\left(n, R^{c}\right)$, we set $N(0)=\left\{i \mid k_{i}(0)=0\right\}$ and write $k_{N(0)}=\left(k_{i_{1}}, \ldots, k_{i_{|N|} \mid}\right) \in J^{r}\left(n, R^{|N|}\right)$ where $i_{1}<\cdots<i_{|N|}$ and $\left\{i_{1}, \ldots, i_{|N|}\right\}=N(0)$. sign $k(0)=\left(\operatorname{sign} k_{1}(0), \ldots, \operatorname{sign} k_{c}(0)\right)$.

Definition 1. A set $C$ in $J^{r}\left(n, R^{p+c}\right)$ is called a rth order condition with $c$ constraints if and only if:

(a) $(h, k) \in C, k, l \in J^{r}\left(n, R^{c}\right)$ with $k_{N(0)}=l_{N(0)}$ and sign $k(0)=\operatorname{sign} l(0)$ imply $(h, l) \in C$.

(b) $C$ is invariant under the group $L^{r}(n)$ of $C^{r}$ diffeomorphisms around $0 \in R^{n}$.

(c) $(h, k) \in C$ implies $(h+b, k) \in C$ for any vector $b \in R^{p}$.

Let $g=\left(g_{1}, \ldots, g_{c}\right)$ be a $C^{\mu}$ constraint on $M^{n}$. For a given point $x \in W_{g}$, a $C^{\mu}(\mu \geqslant r)$ map $f$ from $M^{n}$ into $R^{p}$ is said to satisfy the rth order condition $C$ with constraints $g$ at $x$ if and only if there exists a chart $(U, \varphi)$ around $x$ with $\varphi(x)=0$ such that $j^{r}\left(f \circ \varphi^{-1}, g \circ \varphi^{-1}\right) \in C$. It is clear that whether $f$ satisfies the $r$ th order condition $C$ is independent of the choice of charts $(U, \varphi)$ around $x$ with $\varphi(x)=0$. We remark here that our condition (a) in Definition 1 simply means the right $r$ th order condition should only depend on the $r$-jet of $f$ and the $r$-jet of $W_{g}$ at $x$. When there are no constraints on $M^{n}$, this definition of the $r$ th order condition agrees with my previous definition (cf. [11]).

Now we are ready to state the main results of this paper. One should notice that our results are natural extensions of our previous work [11]. Denote by $C^{\mu}\left(M^{n}, R^{p}\right)$ the space of all $C^{\mu}$ mappings from $M^{n}$ into $R^{p}$ endowed with the Whitney $C^{\mu}$ topology.

MAIN THEOREM. Let $g=\left(g_{1}, \ldots, g_{c}\right)$ be a $C^{\mu}$ constraint on $M^{n}$ satisfying the regularity boundary condition and let $\mu>\min (n, p+c)+1$. Then, there exists a necessary and sufficient $\min (n, p+c)+1$ order algebraic condition for local Pareto optima with the given inequality constraints $g$ for a generic class of mappings in $C^{\mu}\left(M^{n}, R^{p}\right)$. Furthermore, this generic class of mappings can itself be described by $a \min (n, p+c)+1$ order algebraic condition.

Here a $r$ th order condition $C$ in $J^{r}\left(n, R^{p+c}\right)$ is said to be algebraic if and only if the set $C$ is semialgebraic (cf. [11]).

Using standard theorems in stratification theory (cf. [3], [4], [9]), a variant of Thom's transversality theory, together with a study of the first and second order conditions (cf. [10]), yield the following results as a corollary of our Main Theorem.

Structure TheORem. Let $g=\left(g_{1}, \ldots, g_{c}\right)$ be a $C^{\mu}$ constraint on $M^{n}$ satisfying the regularity boundary condition and let $\mu>\min (n, p+c)+2$. 
Then, there exists an open dense subset $\theta$ in $C^{\mu}\left(M^{n}, R^{p}\right)$ such that for each $f$ in $\vartheta$ we have a Whitney prestratification $\mathcal{S}_{f}$ on the feasible set $W_{g}$ with the following properties:

(a) The set $\Theta(f)$ of local Pareto optima is a union of strata in $\mathfrak{S}_{f}$ with dimensions $\leqslant \min (n, p-1)$.

(b) The set $\Theta_{N}(f)$ of all nondegenerate local Pareto optima with constraints $g$ is a union of strata in $\varsigma_{f} . \Theta_{N}(f)$ is dense in $\Theta(f)$.

(c) For $\tilde{f}$ sufficiently close to $f$, there exists a strata-preserving homeomorphism from the pair $\left(\Theta(f), \Theta_{N}(f)\right)$ onto the pair $\left(\Theta(\tilde{f}), \Theta_{N}(\tilde{f})\right)$ provided that $W_{g}$ is compact.

The definition of Whitney prestratification is given in $\$ 3$ (cf. [4]). Those local Pareto optima which can be characterized by the second order criteria as introduced in [10] (cf. definition of $\Theta_{N}$ ) are said to be nondegenerate. Since these criteria are explicitly given and $\Theta_{N}(f)$ is dense in $\Theta(f)$ generically, they are important in applications (cf. [7]). The example of a fold map $f: R^{2} \rightarrow R^{2}$ with constraints $0 \leqslant x \leqslant 1,0 \leqslant y \leqslant 1$ as described earlier exhibits all the generic properties stated in the above Structure Theorem. In fact, for this fold map $f$, one has $\Theta(f)=\Theta_{N}(f)$.

The contents of this paper are as follows: $\$ 2$ contains the definitions of those algebraic conditions in our Main Theorem. It is a natural extension of those algebraic conditions considered in my previous paper [11]. Certainly, the Tarski-Seidenberg theorem on semialgebraic sets and the notion of $v$-sufficiency of jets are important here. In $\$ 3$, we state some basic theorems in stratification theory. A variant of Thom's Transversality Theory, which is suitable for our purposes, is introduced. Combining the facts and the results in $\$ 2$ and $\$ 3$ with the study of the first and second order conditions, we give in the last section the proof of our Main Theorem and Structure Theorem.

Finally, let us point out that the same theorems with simple modifications remain valid if one allows some equality constraints in our optimization problem. (See Remark 6 in \$4.)

2. The algebraic criteria. First, let us state the algebraic criteria $A$ for local Pareto optima with inequality constraints for the class of polynomial mappings. From now on, we identify the jet space $J^{r}\left(n, R^{m}\right)$ with the vector space of polynomial mappings from $R^{n}$ into $R^{m}$ with degree less than or equal to $r$. Thus, the jet space $J^{r}\left(n, R^{p+c}\right)$ is represented by a pair of polynomials maps $(h, k)$ with $h \in J^{r}\left(n, R^{p}\right)$ and $k \in J^{r}\left(n, R^{c}\right)$.

TheORem 1. Let $A=\left\{(z, w) \in J^{r}\left(n, R^{p+c}\right) \mid 0\right.$ is a local Pareto optimum for $z$ with inequality constraints $w \geqslant 0\}$. Then, the subset $A$ is semialgebraic in $J^{r}\left(n, R^{p+c}\right)$.

This theorem is a simple extension of Theorem 1 in [11]. One needs only to 
apply the Tarski-Seidenberg theorem twice as in the proof of Theorem 1 in [11]. Therefore, we leave the details to the reader.

Now let us define the algebraic condition $V_{r}$ which describes the "generic" family of mappings in our Main Theorem. Recall that an $r$-jet $z \in J^{r}\left(n, R^{m}\right)$ is called $v$-sufficient if for any two realizations $f, g$ of $z, f^{-1}(f(0))$ is homeomorphic to $g^{-1}(g(0))$ near 0 . Given an $r$-jet $\zeta=\left(\zeta_{1}, \ldots, \zeta_{m}\right)$ in $J^{r}\left(n, R^{m}\right)$ and a nonempty subset $S=\left\{s_{1}, \ldots, s_{a}\right\} \subset\{1, \ldots, m\}$, set $\zeta_{S}=$ $\left(\zeta_{s_{1}}, \ldots, \zeta_{s_{a}}\right) \in J^{r}\left(n, R^{a}\right)$. For $\omega=\left(\omega_{1}, \ldots, \omega_{c}\right) \in J^{r}\left(n, R^{c}\right)$, let $N=$ $N(\omega(0))=\left\{j \mid \omega_{j}(0)=0\right\} . \omega(0) \geqslant 0$ means $\omega_{j}(0) \geqslant 0$ for all $j$.

Definition 2. Let $V_{r}=\left\{(z, \omega) \in J^{r}\left(n, R^{p+c}\right) \mid \omega(0)\right\} \geqslant 0$ and $\left(z, \omega_{N}\right)_{s}$ is $v$-sufficient for any nonempty subset $S$ in $\{1, \ldots, p+|N|\}$. Denote by $V_{r}^{c}$ the complement of $V_{r}$ in $J^{r}\left(n, R^{p+c}\right)$.

PROPOSITION 1. $V_{r}$ and $V_{r}^{c}$ are algebraic conditions, and the codimension of $V_{r}^{c}$ in $J^{r}\left(n, R^{p+c}\right)$ is greater than or equal to $\max (n-(p+c), 0)+r$.

PROof. It follows from the fact the set of all $r$-jets in $J^{r}\left(n, R^{m}\right)$ which are not $v$-sufficient forms a semialgebraic subset of codimension greater than or equal to $|n-m|+r$ (cf. Theorem 6 in [11]).

The nice thing about this condition $V_{r}$ is the following.

Proposition 2. Let $j^{r}(f, g)=j^{r}(h, k) \in V_{r}$. If 0 is a local Pareto optimum of $f$ with constraints $g \geqslant 0$, then 0 is also a local Pareto optimum of $h$ with constraints $k>0$.

This proposition has been proved in my previous paper (see Theorem 8 in [11]) in the special case that one does not have any constraints on $M^{n}$. One observes that this proposition can be verified from the special case if one gets the next two lemmas.

LeMma 1. Suppose $(f, g)$ is a $C^{r}$ map from a neighborhood $U$ of $0 \in R^{n}$ into $R^{p} \times R^{c}$ with $g(0) \geqslant 0$. Then, 0 is a strict local Pareto optimum of $f$ with inequality constraints $g$ if and only if 0 is a strict local Pareto optimum of $\left(f, g_{N}\right)$ from $U$ into $R^{p} \times R^{|N|}$. Here, $N=\left\{i \mid g_{i}(0)=0\right\}$ and $g(0) \geqslant 0$ means $g_{i}(0) \geqslant 0$ for each $i$.

LeMMA 2. Let $(f, g)$ be a $C^{r}$ map from a neighborhood $U$ of 0 in $R^{n}$ into $R^{p} \times R^{c}$ with $j^{r}(f, g) \in V_{r}$. Then, 0 is a local Pareto optimum of $f$ with constraints $g \geqslant 0$ implies 0 is also a strict local Pareto optimum of $f$ with constraints $g \geq 0$.

Lemma 1 follows easily from the definitions. Thus, it remains to show Lemma 2.

Proof of Lemma 2. Without loss of generality, we assume that $(f, g)(0)=$ 0 . Let $V$ be a small neighborhood of 0 in $U$ such that $D(f, g)_{S}$ is of rank $|S|$ 
over $V \cap\left[(f, g)_{s}^{-1}(0) \backslash\{0\}\right]$ for each nonempty subset $S$ in $\{1, \ldots, p+c\}$. The existence of such a neighborhood is given by the characterization theorem for $v$-sufficiency of jets (cf. Theorem 4 in [11] or Appendix 1 in [1]). Now, suppose this lemma were not valid. Then, there exists a sequence $\left\{y_{n}\right\}$ in $V \backslash\{0\}$ converging to zero such that $f\left(y_{n}\right)=0$ and $g\left(y_{n}\right) \geqslant 0$ for all $n$. For each $n, D\left(f, g_{N\left(y_{n}\right)}\right)$ is of rank $\left|N\left(y_{n}\right)\right|$ at $y_{n}$. Thus, one can choose a sequence $\left\{\tilde{y}_{n}\right\}$ in $U$ such that $\left|\tilde{y}_{n}-y_{n}\right|<1 / n f\left(\tilde{y}_{n}\right)>0$ and $g\left(\tilde{y}_{n}\right)>0$ for any $n$. The fact that this sequence $\left\{\tilde{y}_{n}\right\}$ converges to 0 with $f\left(\tilde{y}_{n}\right)>0=f(0)$ and $g\left(\tilde{y}_{n}\right)>$ 0 for all $n$ contradicts to the assumption that 0 is a local Pareto optimum for $f$ with constraints $g \geqslant 0$. Hence, we finish the proof.

Finally, let us give the algebraic condition $\Theta$ of local Pareto optima in our Main Theorem.

Definition 3. Set $\Theta=A \cap V_{r} \subset J^{r}\left(n, R^{p+c}\right)$. Here $A$ is the semialgebraic set defined in Theorem 1. It follows from Proposition 2 that $\Theta$ is an algebraic condition with $c$ constraints.

Denote by $\mathscr{F}_{g}=\left\{f \in C^{\mu}\left(M^{n}, R^{p}\right) \mid f\right.$ satisfies condition $V_{r}$ with given constraints $g \geqslant 0$ on $W_{g}$ \}. In the next section, we shall develop a variant of Thom's transversality theory which enables us to conclude that $\mathscr{F}_{g}$ is a Baire set in $C^{\mu}\left(M^{n}, R^{p}\right)$ under those assumptions in our Main Theorem.

We finish this section by the following two remarks. The second remark will be used in the remark at the end of $\S 4$.

REMARK 1 . The only properties of the algebraic condition $V_{r}$ needed in this paper are Propositions 1 and 2. Using a construction similar to that used in the proof of Theorem 1 in [8], one can also produce an algebraic condition so that Propositions 1 and 2 hold. Thus, we have another way to choose the algebraic condition $V_{r}$ here.

ReMARK 2. Given $|N| \leqslant \min (n, c)$, set $V_{r}^{*}=\left\{(z, w) \in J^{r}\left(n, R^{p+c}\right) \mid w(0) \geqslant\right.$ $0,|N(w(0))| \leqslant|N|$, and $\left(z, w_{N}\right)_{S}$ is $v$-sufficient for any nonempty subset $S$ in $\{1, \ldots, p+|N(w(0))|\}\}$. If one replaces $V_{r}$ by $V_{r}^{*}$, Proposition 2 remains valid. Proposition 1 becomes that $V_{r}^{*}$ is an algebraic condition with $\operatorname{cod}\left(V_{r}^{*}\right)^{c}$ $\geqslant \max [n-(p+|N|), 0]$.

3. Stratification and transversality. First, we review the relevant parts of stratification theory for semialgebraic sets which are needed in this paper (see [3], [4]).

Let $U, V$ be two $C^{1}$ submanifolds of an open set $W$ in $R^{n}$ with $x \in V$ and $V \subset \bar{U} \backslash U$. We say the triple $(U, V, x)$ satisfies Whitney's condition (b) if for any sequence $\left\{x_{i}\right\}$ in $U$ and sequence $\left\{y_{i}\right\}$ in $V$ such that $x_{i} \rightarrow x, y_{i} \rightarrow x$, $x_{i} \neq y_{i}, \overline{x_{i} y_{i}} \rightarrow l$ (in projective space $P^{n-1}$ ) and $T U_{x_{i}} \rightarrow \tau$ (in the Grassmannian of $(\operatorname{dim} U)$-planes in $\left.R^{n}\right)$, one has $l \subset \tau$. It is not difficult to see that if $\phi$ is a diffeomorphism of $W$ onto an open subset of $R^{n}$, then the triple $(\phi U, \phi V, \phi x)$ satisfies condition (b) if the triple $(U, V, x)$ satisfies condition 
(b). Thus, this condition on $(U, V, x)$ also makes sense when $W$ is a smooth manifold.

DEFINITION 4. Suppose $\mathcal{S}$ is a partition of a subspace $Y$ in a given smooth manifold $X$. The elements in $\delta$ will be called the strata of $\delta$. We say that $\delta$ is a $C^{r}$ Whitney prestratification of $Y$ if the following conditions hold:

(a) Each stratum in $\delta$ is a $C^{r}$ submanifold of $X$.

(b) The family $\delta$ of subsets in $Y$ is locally finite.

(c) If $U$ and $V$ are strata and $\bar{U} \cap V \neq \varnothing$, then $\bar{U} \supset V$.

(d) If $U$ and $V$ are different strata with $\bar{U} \supset V$, then the triple $(U, V, x)$ satisfies Whitney's condition (b) for all $x$ in $V$.

A $C^{s}(s \geqslant 1)$ mapping $f$ from a smooth manifold $M$ into $X$ is said to be transversal to the prestratification $\mathcal{S}$ if $f$ is transversal to all strata of $\mathcal{S}$.

The notion of Whitney prestratification can be regarded as a differentiable version of the $\mathrm{CW}$ complex in the topological category, and is appropriate for developing a transversality theory.

Theorem 2 (Mather, (8.8) Corollary IN [4]). Let $X, M$ be smooth manifolds, $Y$ a closed set in $X, \delta$ a $C^{r}$ Whitney prestratification of $Y$, and $f$ : $M \rightarrow X$ be a $C^{r}(r \geqslant 2)$ mapping transversal to $\mathcal{S}$. Then $f^{*} \mathcal{S}$ is a $C^{r}$ Whitney prestratification of $f^{-1} Y$. Here the family $f^{*} \delta$ consists of connected components of sets of the form $f^{-1} U, U \in \mathcal{S}$.

Now let us state the basic theorem about stratification for semialgebraic sets. The first part of this theorem can be found in [3] or [9]. The second part follows from the proof of the first part easily.

THEOREM 3. Suppose $V_{1}, \ldots, V_{k}$ are semialgebraic subsets in $R^{m}$. Then there exists a smooth Whitney prestratification $\mathcal{S}$ of $R^{m}$ into finitely many semialgebraic strata such that each $V_{i}$ is a union of strata in $\delta$. Furthermore, if $G$ is a set of smooth diffeomorphisms of $R^{m}$ which leaves each $V_{i}$ invariant, then $G$ also leaves the strata of the prestratification $\mathcal{S}$ invariant.

Secondly, we would like to investigate the basic problem about transversal mappings with constraints. Fixing a constraint $g$ in $C^{\mu}\left(M^{n}, R^{c}\right)$ with $\mu>r$, we consider the $C^{\mu-r}$ map $J_{g}^{r}(f): M^{n} \rightarrow J^{r}\left(M^{n}, R^{p+c}\right)$ defined by $J_{g}^{r}(f)(x)=$ $j^{r}(f, g)(x)$, and the $C^{\mu-r}$ map $\Phi_{g}: J^{r}\left(M^{n}, R^{p}\right) \rightarrow J^{r}\left(M^{n}, R^{p+c}\right)$ defined by $\Phi_{g}\left[j^{r}(f)(x)\right]=j^{r}(f, g)(x)$. Here $f$ is any map in $C^{\mu}\left(M^{n}, R^{p}\right), J^{r}\left(M^{n}, R^{m}\right)$ denotes the $r$-jet bundle over $M^{n}$ and $j^{r}(k)(x)$ is the $r$-jet extension of the $C^{\mu}$ map $k: M^{n} \rightarrow R^{m}$ at $x \in M^{n}$ [2]. Clearly, one has $J_{g}^{r}(f)=\Phi_{g} \circ j^{r}(f)$ for any $f$ in $C^{\mu}\left(M^{n}, R^{p}\right)$. Let $A$ be a subset of $\mathcal{G}^{r}\left(M^{n}, R^{p+c}\right)$ with a smooth Whitney prestratification $\mathscr{P}$. Denote by $\mathscr{F}_{g}=\left\{f \in C^{\mu}\left(M^{n}, R^{p}\right) \mid J_{g}^{r} f\right.$ is transversal to $\mathscr{P}\}$. The basic problem here is to describe the set $\mathscr{F}_{g}$. In the case that $\Phi_{g}$ is transversal to $\mathscr{P}$, the set $\mathscr{F}_{g}$ is the same as the set $\mathscr{F}$ of all $f \in C^{\mu}\left(M^{n}, R^{p}\right)$ such that $j^{r} f$ is transversal to $\Phi_{g}^{*}(\mathscr{P})$. For the rest of this section, we shall show 
that $\Phi_{g}$ is transversal to $\mathscr{P}$ under certain nice conditions. Thus, one gets a variant of the transversality theory of Thom.

The jet space $J^{r}\left(M^{n}, R^{m}\right)$ is a smooth fibre bundle over $M^{n}$ with fibre $J^{r}\left(n, R^{m}\right)$ and structure group $L^{r}(n)$. Thus, given a subset $S$ of $J^{r}\left(n, R^{m}\right)$ invariant under the action of $L^{r}(n)$, there exists a unique sub-bundle $S\left(M^{n}, R^{m}\right)$ of $J^{r}\left(M^{n}, R^{m}\right)$ with fibre $S$. It is clear that if $\delta$ is a smooth Whitney prestratification of $S$ with invariant strata under the action of $L^{r}(n)$, then the collection $\delta\left(M^{n}, R^{m}\right)=\left\{U\left(M^{n}, R^{m}\right) \mid U \in \mathcal{S}\right\}$ defines a smooth Whitney prestratification on $S\left(M^{n}, R^{m}\right)$. Denote by $H$ the group of smooth diffeomorphisms $\{\varphi\}$ of $J^{r}\left(n, R^{p+c}\right)$ with the property that for any $(h, k) \in$ $J^{r}\left(n, R^{p+c}\right)$ one has $\varphi(h, k)=(\bar{h}, \bar{k})$ with $\bar{h}=h \bar{k}_{N(0)}=k_{N(0)}$, and sign $\bar{k}=$ sign $k$ (cf. Definition 1). It is clear that the group $H$ leaves any $r$ th order condition with $c$ constraints invariant.

Now, we are ready to state the desired transversality result.

THEOREM 4. Let $g=\left(g_{1}, \ldots, g_{c}\right)$ be a $C^{\mu}$ constraint on $M^{n}$ satisfying the regularity boundary condition introduced in $\S 1$. Suppose $S$ is a subset of $J^{r}\left(n, R^{p+c}\right)$ having a smooth Whitney prestratification $\mathcal{S}$ with invariant strata under the action of $L^{r}(n)$ and $H$. Then, the $C^{\mu-r}(\mu>r)$ map $\Phi_{g}$ is transversal to the smooth Whitney prestratification $\delta\left(M^{n}, R^{p+c}\right)$ of the set $S\left(M^{n}, R^{p+c}\right)$.

In order to prove this theorem, one needs the following:

LemMA 3. The orbit of a given pair $(h, k)$ in $J^{r}\left(n, R^{p+c}\right)$ under the action of $H$ contains an open neighborhood $V$ of $(h, k)$ in the affine subspace $E=\{(\tilde{h}, \tilde{k})$ $\left.\in j^{r}\left(n, R^{p+c}\right) \mid h=\tilde{h}, k_{N(0)}=\tilde{k}_{N(0)}\right\}$.

Proof of Lemma 3. Recall that $N(0)=\left\{i \mid k_{i}(0)=0\right\}$. Let us choose a small open ball $B_{\varepsilon}$ centered at $(h, k)$ so that for any $(\tilde{h}, \tilde{k})$ in $B_{\varepsilon}$ one has $\tilde{k}_{i}(0) \neq 0$ for $i \notin N(0)$. Claim that the open neighborhood $V=B_{\varepsilon} \cap E$ of $(h, k)$ in $E$ has the desired property. Let $(\tilde{h}, \tilde{k})$ belong to $V$. Then, $h=\tilde{h}$ and $k_{N(0)}=\tilde{k}_{N(0)}$. Let $\rho$ be a smooth function from $J^{r}\left(n, R^{p+c}\right)$ into $[0,1]$ with support in $B_{e}$ and $p=1$ on the line segment $J=\{t(h, k)+(1-t)(\tilde{h}, \tilde{k}) \mid 0$ $\leqslant t \leqslant 1\}$. Consider the smooth vector field $\rho \cdot(0, \tilde{k}-k)$. Denote by $\varphi_{1}$ the time-one map of this vector field $\rho \cdot(0, \tilde{k}-k)$. It is easy to see that $\varphi_{1} \in H$ and $\varphi_{1}(h, k)=(\tilde{h}, \tilde{k})$. Hence, we finish the proof of Lemma 3.

It remains to prove Theorem 4. Suppose $\Phi_{g}\left(j^{r} f\right)(p) \in U\left(M^{n}, R^{p+c}\right)$ for some $U$ in $\delta$. Let $(V, \psi)$ be a chart around $p$ such that $\psi(p)=0$. For $x \in \psi(V)$, and $k \in C^{\mu}\left(M^{n}, R^{m}\right)$, set $k_{x}(\xi)=\left(k \circ \psi^{-1}\right)(x+\xi)$. Thus, $k_{x}$ is a $C^{\mu}$ local map from $\left(R^{n}, 0\right)$ into $R^{m}$. The bundles $J^{r}\left(M^{n}, R^{p}\right)$ and $J^{r}\left(M^{n}, R^{p+c}\right)$ over $V$ are trivial. Regarding $\Phi_{g}$ as a $C^{\mu-r}$ map from $\psi(V) \times$ $J^{r}\left(n, R^{p}\right)$ into $\psi(V) \times J^{r}\left(n, R^{p+c}\right)$, one has $\Phi_{g}(x, h)=\left(x, \varphi_{g}(x, h)\right)=$ $\left(x, h, j^{r} g_{x}\right)$. Clearly that $\Phi_{g}$ is transversal to $U\left(M^{n}, R^{p+c}\right)$ at $j^{r} f(p)$ if and only if $\varphi_{g}$ is transversal to $U$ at $\left(0, j^{r}\left(f \circ \psi^{-1}\right)(0)\right)$. Set $N=\left\{i \mid g_{i}(0)=0\right\}$ and 
define $\pi_{N}: J^{r}\left(n, R^{p+c}\right) \rightarrow J^{r}\left(n, R^{|N|}\right)$ by $\pi_{N}(h, k)=\left(h, k_{N}\right)$. By Lemma 3, one knows that $\varphi_{g}$ is transversal to $U$ at $\left(0, j^{r}\left(f \circ \psi^{-1}\right)(0)\right)$ if and only if $\pi_{N} \circ \varphi_{g}$ at $\left(0, j^{r} f(0)\right)$ is transversal to $\pi_{N} \mid U$ at $\left(j^{r} f(0), j^{r} g(0)\right)$. Here $\pi_{N} \mid U$ is the restriction of $\pi_{N}$ on $U$. Define the map $\eta: \psi(V) \rightarrow J^{r}\left(n, R^{|N|}\right)$ by $\eta(x)=j^{r}\left(g_{N}\right)_{x}$ and the map $\rho_{N}: U \rightarrow J^{r}\left(n, R^{|N|}\right)$ by $\rho_{N}(h, k)=k_{N}$. It is easy to see $\pi_{N} \circ \varphi_{g}$ at $\left(0, j^{r} f(0)\right)$ is transversal to $\pi_{N} \mid U$ at $\left(j^{r} f(0), j^{r} g(0)\right)$ if and only if the map $\eta$ at 0 is transversal to $\rho_{N}$ at $\left(j^{r} f(0), j^{r} g(0)\right)$. Recall that the map $g_{N} \circ \psi^{-1}:\left(R^{n}, 0\right) \rightarrow$ $R^{|N|}$ is of rank $|N|$ at 0 . Hence, the $L^{r}(n)$-orbit through $j^{r}\left(g_{N} \circ \psi^{-1}\right)(0)$ is open in $j^{r}(n,|N|)$. The stratum $U$ is invariant under the action of $L^{r}(n)$. These facts plus rank $g_{N} \circ \psi^{-1}=|N|$ at 0 imply that $\eta$ at 0 is transversal to $\rho_{N}$ at $\left(j^{r} f(0), j^{r} g(0)\right)$. Combining with the statement we just established before, we get that $\Phi_{g}$ is transversal to $U\left(M^{n}, R^{p+c}\right)$ at $j^{r} f(p)$. Hence we complete the proof of Theorem 4.

4. The proof and an application of the Main Theorem. Let $g=\left(g_{1}, \ldots, g_{c}\right)$ be a $C^{\mu}$ constraint on $M^{n}$ satisfying the regularity boundary condition introduced in $\S 1$. Denote by $\mathscr{F}_{g}=\left\{f \in C^{\mu}\left(M^{n}, R^{p}\right) \mid f\right.$ satisfies condition $V_{r}$ with the given constraints $g \geqslant 0$ for any $\left.x \in W_{g}\right\}$, where $V_{r}$ is given by Definition 2 with $r=\min (n, p+c)+1$.

Proposition 2 implies that for $f \in \mathscr{F}_{g}$, the algebraic condition $\mathcal{H}$, which is given in Definition 3, is a necessary and sufficient condition for local Pareto optima with the given constraints $g \geqslant 0$. To complete the proof of our Main Theorem, we need to show that $\mathscr{F}_{g}$ is a Baire set in $C^{\mu}\left(M^{n}, R^{p}\right)$. Since $\max (n-(p+c), 0)+r=n+1$. Proposition 1 now says that the complement $V_{r}^{c}$ of $V_{r}$ in $J^{r}\left(n, R^{p+c}\right)$ is a semialgebraic set of codimension $\geqslant n+1$ $(=\max (n-(p+c), 0)+r)$. The semialgebraic set $V_{r}^{c}$ is invariant under the action of $L^{r}(n)$ and $H$. Thus $V_{r}^{c}$ can be written as a finite union of submanifolds $\left\{W_{i}\right\}$ of codimension $\geqslant n+1$ such that each $W_{i}$ is invariant under the action of $L^{r}(n)$ and $H$ (cf. Theorem 3 or [9]). By Theorem 4, $\Phi_{g}$ is transversal to $W_{i}\left(M^{n}, R^{p+c}\right)$ for each $i$. Hence $\Phi_{g}^{-1}\left(V_{r}^{c}\right)$ is the finite union of $C^{\mu-r}$ submanifolds $\Phi_{g}^{-1}\left(W_{i}\left(M^{n}, R^{p+c}\right)\right)$ with codimension $\geqslant n+1$. Therefore, $f \in \mathscr{F}_{g}$ if and only if $j^{r} f$ is transversal to $\Phi_{g}^{-1}\left(W_{i}\left(M^{n}, R^{p+c}\right)\right)$ for all $i$. By Thom's transversality theorem, we conclude that $\mathscr{F}_{g}$ is a Baire set in $C^{\mu}\left(M^{n}, R^{p}\right)$, and finish the proof of our Main Theorem.

Now let us put the first and second order conditions for local Pareto optima, which have been considered in [10] and [7], into our framework. Meanwhile, we shall give more facts about these conditions. Set $\bar{I}_{m}=$ $\left\{\left(y_{1}, \ldots, y_{m}\right) \in R^{m} \mid y_{1} \geqslant 0, \ldots, y_{m} \geqslant 0\right\}$. For a $C^{\mu}$ map $u$ from an open neighborhood $U$ of 0 in $R^{n}$ into $R^{m}, D u(0), D^{2} u(0)$ denote the first and second derivatives of $u$ at 0 .

Definition 5. Set $\theta=\left\{(h, k) \in J^{1}\left(n, R^{p+c}\right) \mid k(0) \geqslant 0\right.$ and there exists $(\lambda, \mu) \in \bar{I}_{p} \times \bar{I}_{c} \backslash\{(0,0)\}$ such that $\lambda \cdot D h(0)+\mu \cdot D k(0)=0$ and $\mu_{i} k_{i}(0)=$ 
0 for all $i$ \}. It is easy to see that $\theta$ is a first order condition with $c$ constraints and that it is equivalent to the first order condition for local Pareto optima with constraints introduced in [10] or [7].

THEOREM 5. $\theta$ is an algebraic condition with c constraints. Furthermore, its codimension in $J^{1}\left(n, R^{p+c}\right)$ is greater than or equal to $\max (n-p+1,0)$.

Proof. The first part follows easily from the Tarski-Seidenberg theorem on semialgebraic sets. For any subset $N$ of $\{1, \ldots, c\}$, set $\theta_{N}=\{(h, k) \in$ $\theta \mid k_{N}(0)=0$ and $k_{i}(0)>0$ for all $\left.i \notin N\right\}$. Clearly, $\theta$ is the disjoint union of all the semialgebraic sets $\left\{\theta_{N}\right\}$. To verify the second part, it suffices to show that the codimension $\theta_{N}$ in $J^{1}\left(n, R^{p+c}\right)$ is greater than or equal to $n-p+1$ for any subset $N$ of $\{1, \ldots, c\}$. The set $\theta_{N}$ is contained in the linear subspace $Y=\left\{(h, k) \in J^{1}\left(n, R^{p+c}\right) \mid k_{N}(0)=0\right\}$ of $J^{1}\left(n, R^{p+c}\right)$. The codimension of $Y$ in $J^{1}\left(n, R^{p+c}\right)$ is equal to $|N|$. Thus, it is enough to show that the codimension of $\theta_{N}$ in $Y$ is greater than or equal to $n-(p+|N|)+1$. Denote by $\pi$ the projection of $Y$ onto $J^{1}(n, p+|N|)$ defined by $\pi(h, k)=(h-h(0)$, $\left.k_{N}\right)$. Set $\Sigma=\left\{z \in J^{1}(n, p+|N|) \mid D z(0)\right.$ is not subjective $\}$. Since the codimension of $\Sigma$ in $J^{1}(n, p+|N|)$ is $\max [n-(p+|N|)+1,0]$ (see [2]), and $\theta_{N} \subset \pi^{-1}(\Sigma)$. One gets that the codimension of $\theta_{N}$ in $Y$ is greater than or equal to $n-(p+|N|)+1$, and completes the proof of the second part of this theorem.

For a $C^{\mu} \operatorname{map}(h, k):\left(R^{n}, 0\right) \rightarrow R^{p} \times R^{c}$, set $K=\left\{v \in R^{n} \mid D h(0)(v) \in \bar{I}_{p}\right.$ and $\left.D k_{N}(0)(v) \in \bar{I}_{|N|}\right\}$.

Definition 6. Set $\Theta_{N}=\left\{(h, k) \in J^{r}\left(n, R^{p+c}\right) \mid k(0) \geqslant 0\right.$, and for each $v \in K \backslash\{0\}$ there exists $(\lambda, \mu) \in \bar{I}_{p} \times \bar{I}_{c} \backslash\{(0,0)\}$ such that $D(\lambda \cdot h+\mu$. $k)(0)=0, \mu_{\mathrm{i}} k_{i}(0)=0$ for all $i$, and $\left.D^{2}(\lambda \cdot h+\mu \cdot k)(0)(v)<0\right\}$.

Definition 7. Set $\tilde{\Theta}=\left\{(h, k) \in J^{r}\left(n, R^{p+c}\right) \mid k(0) \geqslant 0\right.$, and for each $v \in$ $K \backslash\{0\}$ there exists $(\lambda, \mu) \in \bar{I}_{p} \times \bar{I}_{c} \backslash\{(0,0)\}$ such that $D(\lambda \cdot h+\mu \cdot k)(0)=$ $0, \mu_{i} k_{i}(0)=0$ for all $i$, and $\left.D^{2}(\lambda \cdot h+\mu \cdot k)(0)(v) \leqslant 0\right\}$.

It is not difficult to see that $\Theta_{N}$ and $\tilde{\Theta}$ are second order conditions and that they are equivalent to those second order conditions for local Pareto optima with constraints introduced in [10] or [7]. Let $f \in C^{\mu}\left(M^{n}, R^{p}\right)$, $g \in C^{\mu}\left(M^{n}, R^{c}\right)$ and $x \in M^{n}$. If the mapping $(f, g)$ satisfies condition $\Theta_{N}$ at $x$ then $x$ is a local Pareto optimum of $f$ on $W_{g}$, and this point $x \in M^{n}$ will be called a nondegenerate local Pareto optimum of $f$ with constraints $g \geqslant 0$. If $x \in M^{n}$ is a local Pareto optimum of $f$ on $W_{g}$, then $f$ satisfies the condition $\tilde{\Theta}$ with constraints $g$ at $x$.

THEOREM 6. $\Theta_{N}$ and $\tilde{\Theta}$ are second order algebraic conditions with constraints. The set $\Theta_{N}$ is dense in $\tilde{\Theta}$.

Proof. We need to show that $\Theta_{N}$ are $\tilde{\Theta}$ are semialgebraic sets in $J^{r}\left(n, R^{p+c}\right)$. Set $A_{N}=\left\{(h, k, v) \in J^{r}\left(n, R^{p+c}\right) \times R^{n} \mid\right.$ there exists $(\lambda, \mu) \in \bar{I}_{p}$ 
$\times \bar{I}_{c} \backslash\{(0,0)\}$ such that $D(\lambda \cdot h+\mu \cdot k)(0)=0, \mu_{i} k_{i}(0)=0$ for all $i$, and $\left.D^{2}(\lambda \cdot h+\mu \cdot k)(0)(v)<0\right\}$. Set $\tilde{A}=\left\{(h, k, v) \in J^{r}\left(n, R^{p+c}\right) \times R^{n} \mid\right.$ there exists $(\lambda, \mu) \in \bar{I}_{p} \times \bar{I}_{c} \backslash\{(0,0)\}$ such that $D(\lambda \cdot h+\mu \cdot k)(0)=0, \mu_{i} k_{i}(0)=0$ for all $i$, and $\left.D^{2}(\lambda \cdot h+\mu \cdot k)(0)(v) \leqslant 0\right\}$. Set also $B=\{(h, k, v) \in$ $\left.J^{r}\left(n, R^{p+c}\right) \times R^{n} \mid v \in K \backslash\{0\}\right\}$ and $W=\{(h, k) \mid k(0) \geqslant 0\}$. The sets $B$ and $W$ are semialgebraic by definition. Using Tarski-Seidenberg Theorem, we know that $A_{N}$ and $\tilde{A}$ are semialgebraic sets. Denote by $\pi$ the projection from $J^{r}\left(n, R^{p+c}\right) \times R^{n}$ onto its first factor. One has $\Theta_{N}^{c}=W^{c} \cup \pi\left[B \cap A_{N}^{c}\right]$ and $\tilde{\Theta}^{c}=W^{c} \cup \pi\left[B \cap \tilde{A}^{c}\right]$. By Tarski-Seidenberg Theorem again and the fact that the complement of a semialgebraic set is a semialgebraic set, we conclude that both $\Theta_{N}$ and $\tilde{\Theta}$ are semialgebraic.

It remains to prove that $\Theta_{N}$ is dense in $\tilde{\Theta}$. Let $q(x)$ be a negative definite quadratic form on $R^{n}$. Thus $D^{2} q(0)(v)<0$ for all $v \in R^{n} \backslash\{0\}$. Set $Q=$ $(q, \ldots, q) \in J^{r}\left(n, R^{p+c}\right)$. Let $(h, k)$ be a typical point in $\tilde{\Theta}$. Set $\left(h_{\varepsilon}, k_{\varepsilon}\right)=$ $(h, k)-\varepsilon^{2} Q$ for any real number $\varepsilon$. Clearly $\left(h_{\varepsilon}, k_{\varepsilon}\right) \in \tilde{\Theta}_{N}$ and $\left(h_{\varepsilon}, k_{\varepsilon}\right) \rightarrow(h, k)$ as $\varepsilon \rightarrow 0$. Thus, the set $\Theta_{N}$ is dense in $\tilde{\Theta}$, and we complete the proof of Theorem 6. Obviously, the set $\theta$ and $\tilde{\Theta}$ are closed in $J^{1}$ and $J^{2}$ respectively. However, this fact is not needed here.

Finally, we give the proof of our Structure Theorem. The notation that will be used is the same as in that theorem. Let $\Theta, V$ be two algebraic conditions with constraints given in our Main Theorem. Thus, the codimension of $V^{C}$ is greater than or equal to $n+1$ and for any $f$ satisfying condition $V$, a point $x$ in $M^{n}$ is a local Pareto optimum for $f$ on $W_{g}$ if and only if $f$ satisfies condition $\Theta$ at $x$. (These conditions $\Theta, V$ are not necessarily the same as given in the proof of our Main Theorem.) Set $r=\min (n, p)+1$. Denote by $\pi_{r, t}$ : $J^{r}\left(n, R^{m}\right) \rightarrow J^{t}\left(n, R^{m}\right)$ the projection defined by $\pi_{r, t}\left(j^{r} f\right)=j^{t} f$. From Theorem 3, one can find a smooth Whitney prestratification $\delta$ of $J^{r}\left(n, R^{p+c}\right)$ such that each one of the semialgebraic sets $\pi_{r, 2}^{-1}\left(\Theta_{N}\right), \pi_{r, 2}^{-1}(\Theta), \pi_{r, 0}^{-1}\left(R^{p} \times \bar{I}_{c}\right)$ is a finite union of strata in $\mathcal{S}$ and each stratum in $\mathcal{S}$ is invariant under $L^{r}(n)$ and $H$. From Theorem 4, we know that the $C^{\mu-r}$ map is transversal to the smooth Whitney prestratification $\delta\left(M^{n}, R^{p+c}\right)$. Thus, by Theorem 2 $\Phi_{g}^{*}\left(\delta\left(M^{n}, R^{p+c}\right)\right)$ is a $C^{\mu-r}$ Whitney prestratification of $J^{r}\left(M^{n}, R^{p}\right)$. Denote by $\theta$ the class of $C^{\mu}$ maps $f$ in $C^{\mu}\left(M^{n}, R^{p}\right)$ which are transversal to the $C^{\mu-r}$ Whitney prestratification $\Phi_{g}^{*}\left(\delta\left(M^{n}, R^{p+c}\right)\right)$. Thom's transversality theorem implies that $\mathcal{O}$ is an open dense subset of the space $C^{\mu}\left(M^{n}, R^{p}\right)$ endowed with the fine $C^{\mu}$ topology. Claim that this open dense subset $\theta$ in $C^{\mu}\left(M^{n}, R^{p}\right)$ has the desired property described in the Structure Theorem. Let $f \in \mathcal{O}$. It is clear from the construction that $H_{N}(f), \Theta(f), W_{g}$ are union of strata in the $C^{\mu-r}$ Whitney prestratification $f^{*}\left(\Phi_{g}^{*}\left(\delta\left(M^{n}, R^{p+c}\right)\right)\right)$. Let $\delta_{f}$ be the restriction of this prestratification on $W_{g}$. Thus, $\Theta_{N}(f)$ and $\Theta(f)$ are union of strata in $\delta_{f}$. For $\pi_{r, 2}^{-1}(\Theta) \subset \pi_{r, 1}^{-1}(\theta)$ and $\operatorname{cod} \pi_{r, 1}^{-1}(\theta) \geqslant \max (n-p+$ $1,0)$ (Theorem 5), one gets that the strata of $\delta_{f}$ in $\Theta(f)$ are of dimension $\leqslant$ 
$\min (n, p-1)$. From Theorem 6 and $\Theta \subset \tilde{\Theta}$, we know that $\Theta_{N}$ is dense in $\Theta$. Hence, $\Theta_{N}(f)$ is dense in $\Theta(f)$. Here, one uses the fact that: Suppose a map $\varphi: M \rightarrow N$ is transversal to a closed subset $A$ in $N$ with Whitney prestratification $\mathscr{P}$ on $A$, and a set $B$ in $N$ which is a union of strata in $\mathscr{P}$. Then, $B$ is dense in $A$ implies that $\varphi^{-1}(B)$ is dense in $\varphi^{-1}(A)$. The last assertion follows from Thom's first isotopy lemma [4]. Therefore, we finish the proof of our Structure Theorem.

Let us conclude this paper with several remarks.

REMARK 3. Suppose $x$ is a local maximum for the restriction of the real valued function $\lambda_{1} f_{1}+\cdots+\lambda_{p} f_{p}$ on the feasible set $W_{g}$ with $\lambda_{1}>$ $0, \ldots, \lambda_{p}>0$. Then, $x$ is also a local Pareto optimum of $f$ with the given constraints $g \geqslant 0$. Therefore, $W_{g}$ nonempty and compact implies that the set $\Theta(f)$ of local Pareto optima with constraints is nonempty.

REMARK 4. The dimension of $\Theta(f)$ in the conclusion of our Structure Theorem can be zero. For example, let $M^{n}=R^{p}=R^{2}, g(x, y)=(1-x$, $x+1,1-y, y+1)$ and $f(x, y)=(2 x+y, x+2 y)$. The image $f\left(W_{g}\right)$ is the parallelogram with vertices $( \pm 3, \pm 3),( \pm 1, \mp 1)$, and $\Theta(f)=\{(1,1)\}$.

REMARK 5. Set $|N|=\max \left\{|N(x)| \mid x \in W_{g}\right\}$, where $N(x)=\left\{i \mid g_{i}(x)=0\right\}$. Our Main Theorem and Structure Theorem are still valid when one replaces $\min (n, p+c)+1, \min (n, p+c)+2$ by the (possibly) smaller numbers $\min (n, p+|N|)+1, \min (n, p+|N|)+2$ respectively. For the proof, it suffices to substitute $V_{r}$ by $V_{r}^{*}$, which has been defined in Remark 1 at the end at $\$ 2$.

REMARK 6. If one considers the constraints $g_{1}=0, \ldots, g_{m}=0, g_{m+1} \geqslant$ $0, \ldots, g_{c} \geqslant 0$ instead of the constraints $g_{1} \geqslant 0, \ldots, g_{c} \geqslant 0$ in our optimization problem. The Main Theorem and Structure Theorem remain valid provided the numbers $\min (n, p+c)+1, \min (n, p+c)+2$ and $\min (n, p-$ 1) appearing in those theorems are replaced by $\min (n-m, p+c-m)+1$, $\min (n-m, p+c-m)+2$ and $\min (n-m, p-1)$ respectively. Similar proofs still work. However, they are more delicate.

\section{REFERENCES}

1. T. C. Kuo, Characterizations of v-sufficiency of jets, Topology 11 (1972), 115-131.

2. H. I. Levine, Singularities of differentiable mappings, Proceedings of the Liverpool Singularities Symposium. I, Lecture Notes in Math., vol. 192, Springer, New York and Berlin, 1971.

3. S. Lojasiewicz, Ensembles semi-analytiques, Preprint, Inst. Hautes Études Sci. Publ. Math.

4. J. N. Mather, Stratifications and mappings, Dynamical Systems (Proc. Sympos., Univ. of Bahia, Salvador, 1971), Academic Press, New York, 1973.

5. C. Morlet, Le lemme de Thom et les théorèmes de plongement de Whitney, Séminaire $\mathrm{H}$. Cartan (1961-1962), Secrétariat math., Paris, 1964.

6. S. Smale, Global analysis and economics. I. Pareto optimum and a generalization of Morse theory, Dynamical Systems (Proc. Sympos., Univ. of Bahia, Salvador, 1971), Academic Press, New York, 1973, pp. 531-544. 
7. 3 (1974).

, Global analysis and economics. V: Pareto theory with constraints, J. Math. Econom.

8. R. Thom, Local topological properties of differentiable mappings, Differential Analysis, Oxford Univ. Press, 1964, pp. 191-202.

9. C. T. C. Wall, Regular stratifications, Dynamical Systems, Warwick, 1974.

10. Y. H. Wan, On local Pareto optima, J. Math. Econom. 2 (1975), 35-42.

11. __ On the algebraic criteria for local Pareto optima. I, Topology 16 (1977), 113-117.

12. __ Remarks on local Pareto optima, preprint.

Department of Mathematics, State University of New York at Buffalo, Buffalo, New YoRK 14214 